\title{
Radiotherapy strategies for recurrent and metastatic breast cancer
}

\author{
Kinji Nishiyama
}

Published online: 6 September 2011

(C) The Japanese Breast Cancer Society 2011

Treatment for breast cancer is multidisciplinary. Radiotherapy (RT) is applied routinely after partial mastectomy. In the journal Breast Cancer a topic on accelerated breast irradiation was addressed as a special feature entitled New Trends in Radiation Therapy. RT for bone metastasis or brain metastasis accounts for more than $10 \%$ of irradiated cases of breast cancer. For breast cancer with long-term prognosis, comprehensive understanding of the optimum RT for various conditions is mandatory. This is why we publish three articles on radiotherapy strategies for recurrent and metastatic breast cancer in this issue.

Bone metastasis, which is the most frequent metastatic site in all malignancy, accounts for $70 \%$ of all metastases from breast cancer. Although bone metastasis is often observed at diagnosis in lung cancer and prostate cancer, it is detected as recurrence after initial treatment of breast cancer. Survival after bone metastasis is approximately 6 months for patients with lung cancer. Under the corresponding conditions for breast cancer, however, survival is much longer-five-year survival after detection of bone metastasis exceeds 50\% (experience at Osaka Medical Center for Cancer and Cardiovascular Diseases-OMCC). Although external radiotherapy had been mainstay for bone metastasis, bisphosphonate has emerged as the first-line treatment for bone metastasis from breast cancer. RT has been indicated for cases that are refractory to bisphosphonate, and achieves 5-year survival of $18.4 \%$ after RT (OMCC). Recently ${ }^{89} \mathrm{Sr}$ chloride has been indicated for multiple bone metastases.

K. Nishiyama $(\bowtie)$

Osaka Medical Center for Cancer and Cardiovascular Diseases,

1-3-3 Nakamichi, Higashinari-ku, Osaka 537-8511, Japan

e-mail: nisiyama-ki@mc.pref.osaka.jp
From 2001-2005 OMCC recorded 178 and 43 cases of brain metastases (BM) from lung cancer and breast cancer, respectively. Thus, breast cancer is the second leading origin of BM after lung cancer. Median survival for BM from lung cancer and breast cancer was 6 and 9 months, respectively. The small difference in prognosis for BM compared with metastasis is attributed to a larger proportion of RPA III in BM from breast cancer (Aoyama). In addition to surgical resection, there are two radiotherapy approaches to BM, whole-brain radiotherapy (WBRT) and stereotactic radiosurgery (SRS). Addition of focal aggressive treatment (surgical resection or SRS) to WBRT can improve local control in the brain. However, it does not generally affect the prognosis of BM except for solitary $\mathrm{BM}$. Aoyama proposes use of SRS as a treatment option in cases in which scheduled monitoring of the brain tumor is possible.

Loco-regional recurrence is also a crucial issue for breast cancer with long-term prognosis. Mastectomy is a mainstay for ipsilateral breast recurrence after breast conservative therapy (Shikama). Because of superior results, treatment for isolated axillary and supraclavicular is also surgical resection after careful examination of the breast. Data on the role of systemic therapy for regional recurrence are few and controversial, although chemotherapeutic regimens including anthracycline or taxane are recommended. There is a trend towards administering systemic therapy for supraclavicular lymph node recurrence (Shikama). The role of radiotherapy for regional lymph nodes to improve prognosis should be examined. 